\title{
Raman spectrometry study of phonon anharmonicity of hafnia at elevated temperatures
}

\author{
Chen W. Li, * Michael M. McKerns, and B. Fultz \\ Materials Science, California Institute of Technology, Pasadena, California 91125, USA \\ (Received 12 March 2009; revised manuscript received 9 June 2009; published 27 August 2009)
}

\begin{abstract}
Raman spectra of monoclinic hafnium oxide $\left(\mathrm{HfO}_{2}\right)$ were measured at temperatures up to $1100 \mathrm{~K}$. Raman peak shifts and broadenings are reported. Phonon dynamics calculations were performed with the shell model to obtain the total and partial phonon density of states, and to identify the individual motions of Hf and $\mathrm{O}$ atoms in the Raman modes. Correlating these motions to the thermal peak shifts and broadenings, it was found that modes involving changes in oxygen-oxygen bond length were the most anharmonic. The hafniumdominated modes were more quasiharmonic and showed less broadening with temperature. Comparatively, the oxygen-dominated modes were more influenced by the cubic term in the interatomic potential than the hafnium-dominated modes. An approximately quadratic correlation was found between phonon-line broadening and softening.
\end{abstract}

DOI: 10.1103/PhysRevB.80.054304

PACS number(s): 63.20.-e, 77.84.Bw, 61.66.-f, 78.20.Bh

\section{INTRODUCTION}

Hafnium oxide $\left(\mathrm{HfO}_{2}\right)$, also known as hafnia, is one of the most common and stable compounds of hafnium found in nature. Hafnium-based oxides are leading candidates to replace silicon oxide as gate insulators in field-effect transistors, largely because the high dielectric constant of hafnia allows greater miniaturization of microelectronic components without increased leakage current. ${ }^{1,2}$ Owing to its chemical stability, physical hardness, high refractive index, and high threshold for laser damage, hafnia has been widely used in coatings on optical components for laser applications in the near UV to IR spectral regions. Hafnia is also an important material for applications at high temperatures owning to its high bulk modulus and high melting temperature. ${ }^{3}$

Hafnia and zirconia $\left(\mathrm{ZrO}_{2}\right)$ are monoclinic $\left(P 2_{1} c\right)$ at ambient pressure and temperature. Both hafnia and zirconia transform to a tetragonal phase $\left(\mathrm{P}_{2} n m c\right)$ at approximately $2000 \mathrm{~K},{ }^{4,5}$ and to a cubic phase $(F m 3 m)$ at higher temperatures and pressures. ${ }^{4}$ Many other similarities between hafnia and zirconia are sometimes attributed to the lanthanide contraction $^{5}$ and these include a nearly one-to-one correspondence of their Raman spectra. 5,6 The room-temperature monoclinic crystal structure of hafnia has four formula units (i.e., 12 atoms) per unit cell and hence 36 normal modes at the $\Gamma$ point. The group theoretical analysis of mode symmetry by Anastassakis et al. $^{7}$ predicts 18 Raman-active modes $\left(A_{g}\right.$ and $\left.B_{g}\right), 15$ infrared-active modes, and 3 acoustic modes. The Raman-active and infrared-active modes of hafnia prove mutually exclusive (a mode can be Raman-active only or IR-active only). Prior experimental and theoretical Raman spectroscopy analysis of hafnia has been hindered by the complexity of the vibrational symmetry. Anastassakis et al. ${ }^{7}$ were able to identify 17 modes of vibration, but assignment of the peaks proved difficult, especially for modes at higher energies. Other studies have focused on the phase transition under pressure ${ }^{8,9}$ while first-principles calculations were performed to find obtain a better understanding of the vibration modes. ${ }^{10,11}$ To date the assignment of Raman peaks of hafnia has not been fully convincing.
The focus of the present work is on the nonharmonic vibrational dynamics of hafnia. High-temperature vibrational studies of metal oxides are relatively rare. Several metal oxides, such as beryllia $(\mathrm{BeO})$ and lithia $\left(\mathrm{Li}_{2} \mathrm{O}\right)$, have been shown to exhibit anharmonic behavior at elevated temperatures, primarily due to a change in phonon lifetime resulting from phonon-phonon interactions. ${ }^{12}$ Although there have been no previous reports of phonon anharmonicity in hafnia, its unusually high specific heat at temperatures above 800 $\mathrm{K},{ }^{13}$ which is well below the temperature of any phase transition, suggests interesting anharmonic behavior. Similar behavior has been reported in a thermodynamic assessment of zirconia. ${ }^{14} \mathrm{~A}$ contribution to the heat capacity at constant pressure is expected as a crystal expands against its bulk modulus. "Quasiharmonic" shifts of phonon frequencies arise because these frequencies are reduced in an expanded crystal, thus yielding a larger vibrational entropy. The reported increase in heat capacity $^{13}$ of around 2-3 $\times 10^{-2}[\mathrm{~J} /(\mathrm{mol} \mathrm{K})] / \mathrm{K}$ is much larger than expected from this quasiharmonic phonon mechanism, however. Anharmonic effects from phonon-phonon interactions are therefore of interest. Inelastic neutron-scattering studies of hafnia are impractical owing to the large neutron absorption cross section of hafnium. ${ }^{15}$ Previous temperature-dependent Raman spectroscopy studies of hafnia were limited to $800 \mathrm{~K},{ }^{9}$ and were performed with relatively low resolution. Besides the phonon frequency-shift information that is typically used to understand vibrational thermodynamics, line broadening information from high-resolution measurements allows an assessment of how anharmonic effects originate from the thirdorder or fourth-order terms of the interatomic potential. In the present work on hafnia, we report measurements of Raman line positions and shapes to temperatures of $1100 \mathrm{~K}$, characterize the types of modes that are most anharmonic and correlate anharmonic effects to the vibrational displacements of individual atoms in the unit cell.

\section{EXPERIMENT}

Samples were prepared from commercial hafnia powder ${ }^{16}$ having a grain size around $40 \mu \mathrm{m}$. Two methods of prepa- 
ration were used, (1) hafnia powder was loosely packed inside a quartz cell and (2) powder was compressed into pellets at ambient temperature and under a pressure of $30 \mathrm{MPa}$. Both samples were characterized by $\mathrm{x}$-ray diffractometry and both types exhibited a pure monoclinic phase (with phase fraction of at least $99 \%$ ). It was found that samples prepared as pellets gave spectra identical to those prepared as a loose powder. The samples were mounted in a quartz optical cell of $1 \mathrm{~mm}$ thickness, evacuated by a turbomolecular pump to a pressure below $10^{-8} \mathrm{~Pa}$. Sample heating was provided by electrical resistance heating elements in the vacuum space. The furnace was composed of ceramic rods for insulation, stainless steel flanges as structure support, several layers of niobium foil as heat shields, stainless steel plates, and aluminum foil covering the interior and exterior of the quartz tube as radiation shielding. A temperature controller was used to drive a $1 \mathrm{~kW}$ dc power supply through a solid-state relay. Uniformity of sample temperature was confirmed by placing multiple ultrafine thermocouples at different positions inside the quartz sample cell.

The excitation source for the Raman spectrometer was the $532 \mathrm{~nm}$ line of a Nd:YAG laser ${ }^{17}$ at power levels of $200 \mathrm{~mW}$ or lower. A high-efficiency edge filter ${ }^{18}(\mathrm{OD}=6)$ was used to block the laser line. A single pass spectrometer ${ }^{19}$ was used for the spectral imaging on a two-dimensional chargecoupled device camera with thermoelectric cooling. ${ }^{20}$ Raman spectra were acquired at temperatures from 300 to $1100 \mathrm{~K}$ in increments of approximately $25 \mathrm{~K}$, with a temperature resolution of $\pm 1 \mathrm{~K}$. Spectra at the higher temperatures had a sloping background from blackbody radiation that was subtracted before fitting. Measurements at $300 \mathrm{~K}$, repeated after the heating sequence, confirmed that the samples underwent no changes that affected their Raman spectra.

\section{RESULTS}

The Raman spectrum at room temperature and ambient pressure appears to consist of 17 peaks but the second peak consists of two overlapping modes (labeled 2 and 3 in Fig. 1). Spectra measured at temperatures above $600 \mathrm{~K}$ show the separation of a shoulder from the second peak, evidence of a two-peak structure. At higher temperatures, several of the weaker modes either could not be resolved from the background or their position could not be determined reliably. Nevertheless, several strong peaks remained distinct to the highest temperature attained. The spectra acquired at all 33 temperatures (many not shown in Fig. 1) were used to obtain the effects of temperature on line position and linewidth. A curved background, believed intrinsic to each sample, was first subtracted from each spectrum. Each measured spectrum was then fit with multiple Lorentzian functions to extract the centroid and the full width at half maximum for each peak. The linewidth of the spectrometer resolution was negligible when compared to the error in the fitted linewidth.

The results, presented in Figs. 2 and 3, show that with increasing temperature, the Raman peaks generally shift to lower frequencies, and generally broaden in width. (Owing to the larger uncertainty for the linewidths of weak peaks, results from only the stronger peaks are presented in Fig. 3.)
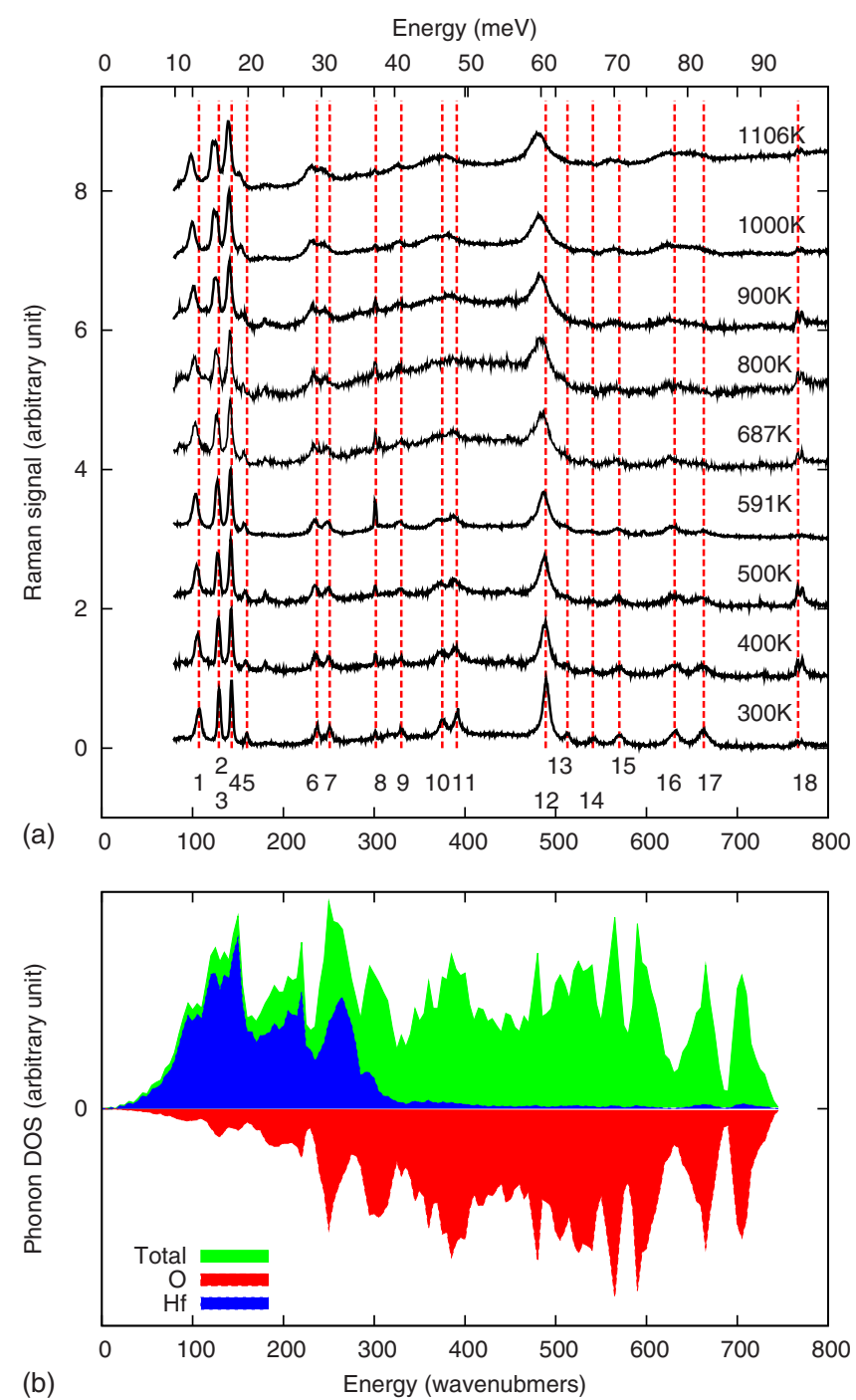

FIG. 1. (Color online) (a) Raman spectra of hafnia at temperatures from 300 to $1106 \mathrm{~K}$, with laser power at $200 \mathrm{~mW}$, exposure time of $30 \mathrm{~s}$ and at least 10 accumulations of the spectra. Peaks are numbered for reference. The features between peaks 5 and 6 are artifacts. (b) Partial and total phonon density of states of hafnia at room temperature, calculated using GULP. Hafnium contributes much more strongly to the lower-energy modes while oxygen contributes to the higher-energy modes.

There are substantial differences in the amount of shift and amount of broadening for the different Raman peaks, with some peaks showing minimal effects of temperature. Interpreting the different nonharmonic behaviors of the different Raman peaks requires a detailed understanding of the atom motions in the different Raman modes.

\section{CALCUlations}

To assign vibration modes to spectral peaks, Raman mode frequency calculations were performed with the GULP package, ${ }^{21}$ using the force-field constants from Lewis, ${ }^{22}$ and the Buckingham two-body shell model potential. Results are listed in Table I. Good agreement was found between the 


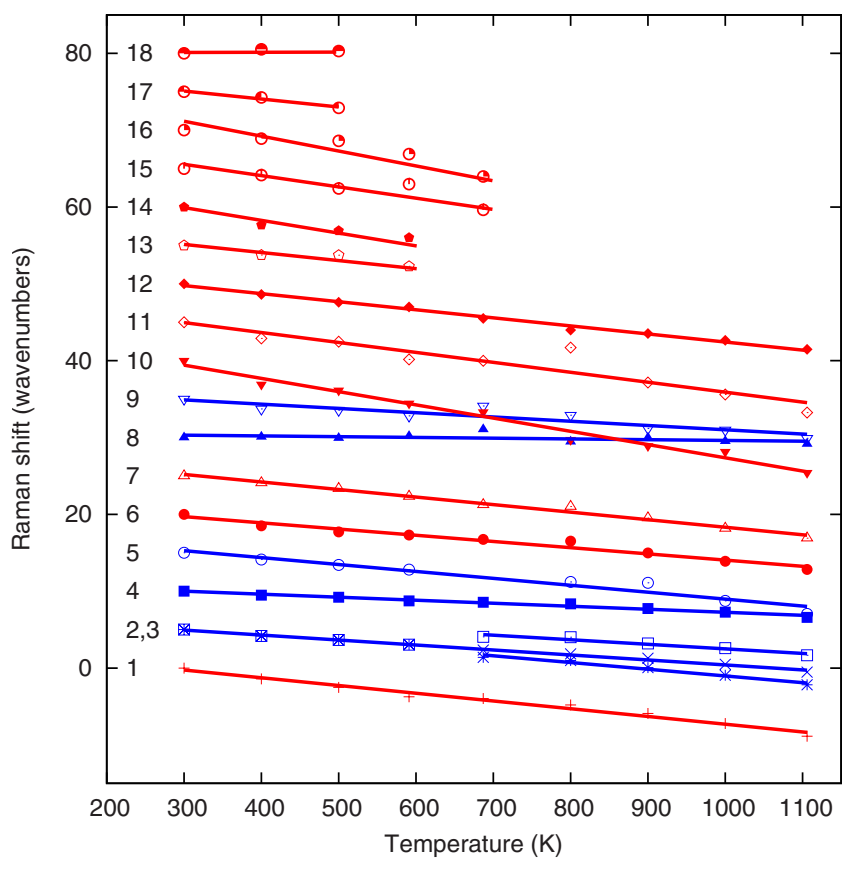

FIG. 2. (Color online) Temperature-dependent Raman mode softening. At $300 \mathrm{~K}$, modes are evenly spaced in the figure to facilitate comparison of thermal shifts. Blue (dark gray) and red (light gray) represent metal and oxygen-dominated modes, respectively. Lines are linear fits to the data points. Note that second and third modes are decoupled above $700 \mathrm{~K}$, where the line between the 2 nd and 3rd modes represent an average peak position. Most error bars are negligibly small. Peaks from the 13th to 18th were not sufficiently distinct to allow fitting at high temperature.

calculations and Raman spectroscopic data from the present and previous experiments and reasonable agreement with previous $a b$ initio calculations, especially with the generalized gradient approximation (GGA) method.

Calculations of the phonon density of states (DOS) were also performed with the GULP software package by sampling over points in $k$ space. The resulting total and partial DOS are shown in Fig. 1. Many of the phonon branches had low dispersion, giving a number of sharp peaks in the DOS. The

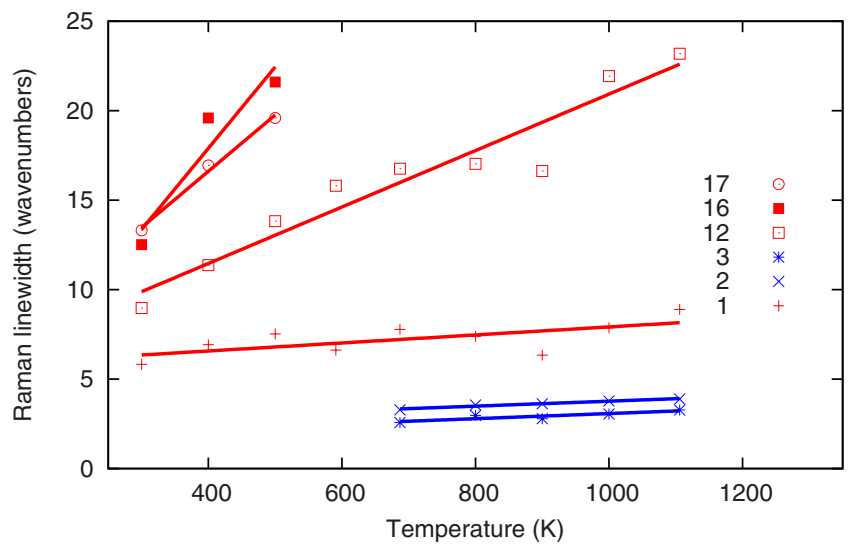

FIG. 3. (Color online) Temperature-dependent peak broadening. Blue (dark gray) and red (light gray) represent metal- and oxygendominated modes, respectively. Lines are linear fits to the data. maximum phonon energy was about $90 \mathrm{meV}$. Calculations with GULP of the heat capacity at constant volume gave results as expected for harmonic oscillators, approaching 25 $\mathrm{J} /(\mathrm{mol} \mathrm{K})$ at temperatures above $1000 \mathrm{~K}$. The eigenvectors of phonons at the $\Gamma$ point were used to estimate the participation of $\mathrm{Hf}$ or $\mathrm{O}$ in each Raman-active mode, with results listed in Table I. Consistent with Fig. 1, the heavier metal atoms dominate the low-energy modes whereas the lighter oxygen atoms have most of the energy of the high-energy modes. Most of the Raman modes are either metal dominated $(2,4,5$, and 8$)$, or oxygen dominated $(6,7,10$, and 12-18). The few mixed modes (modes 1, 3, 9, and 11) are still heavily biased. This approximate separation of modes into $\mathrm{Hf}$ modes and $\mathrm{O}$ modes is consistent with the large difference in mass of oxygen and hafnium atoms.

\section{DISCUSSION}

The quasiharmonic softening of a normal mode is related to thermal expansion

$$
\omega_{j}(T)=\omega_{j}(0) \exp \left[-\gamma_{j} \int_{0}^{T} \beta(T) d T\right],
$$

where $\omega_{j}(T)$ is a temperature-dependent vibrational frequency, $\beta$ is the volume thermal expansivity, and $\gamma_{j}$ is the mode Grüneisen parameter, which relates the fractional change in vibrational frequency to the fractional change in volume

$$
\frac{\Delta \omega_{j}}{\omega_{j}}=-\gamma_{j} \frac{\Delta V}{V} .
$$

The thermodynamic Grüneisen parameter, $\gamma$, for a quasiharmonic solid is

$$
\gamma=\frac{\beta B V}{C_{V}},
$$

where $B$ is bulk modulus and and $C_{V}$ is the specific heat at constant volume. This $\gamma$ is an average of all mode Grüneisen parameters $\left\{\gamma_{j}\right\}$, weighted by the modal Einstein specific heats $C_{j}$

$$
\gamma=\frac{\sum_{j} \gamma_{j} C_{j}}{\sum_{j} C_{j}} .
$$

From Table I, however, we see that an average Grüneisen parameter misses the rich variation in the mode Grüneisen parameters. Such a wide variation in mode Grüneisen parameters might be expected from the highly anisotropic thermal expansion of hafnia. From 300 to $500 \mathrm{~K}$, its linear thermal expansion is $0.23 \%, 0.15 \%$, and $0.0 \%$ for the $c, a$, and $b$ axes, respectively. At higher temperatures, the $a$ and $c$ axes still expand much faster than the $b$ axis. ${ }^{23}$

The remainder of this paper focuses on the nonharmonicity of individual modes. Figure 2 and Table I show that temperature-dependent frequency shifts do not depend on the energy of each normal mode, as might be expected if all $\gamma_{j}$ of Eq. (2) were similar. There is, instead, a tendency of the 
TABLE I. Raman mode frequencies, fractional atomic contribution for each mode, and mode Grüneien parameters.

\begin{tabular}{cccccccccc}
\hline \hline Mode & Exp & GULP & Lt1 & Lt2 & Lt3 & Hf\% & O\% & $\gamma_{j}^{\mathrm{d}}$ \\
\hline 1 & 106.8 & 120.9 & 128 & 125 & 113 & 0.30 & 0.70 & 4.83 \\
2 & 129.1 & 162.5 & 142 & 132 & 133 & 0.95 & 0.05 & 3.45 \\
3 & 129.1 & 172.8 & 131 & 120 & 133 & 0.74 & 0.26 & 2.35 \\
4 & 142.9 & 181.0 & 152 & 171 & 149 & 0.99 & 0.01 & 1.40 \\
5 & 159.6 & 185.8 & 175 & 152 & 164 & 0.99 & 0.01 & 2.8 \\
6 & 236.8 & 228.2 & 250 & 223 & 242 & 0.05 & 0.95 & 1.75 \\
7 & 251.3 & 268.4 & 261 & 248 & 256 & 0.03 & 0.97 & 2.00 \\
8 & 301.5 & 291.8 & 326 & 339 & 323 & 0.97 & 0.03 & 0.17 \\
9 & 329.9 & 308.3 & 380 & 318 & 336 & 0.81 & 0.19 & 0.4 \\
10 & 375.0 & 341.2 & 423 & 382 & 382 & 0.10 & 0.90 & 0.8 \\
11 & 391.1 & 357.2 & 424 & 385 & 398 & 0.13 & 0.87 & 1.8 \\
12 & 489.3 & 448.1 & 514 & 440 & 498 & 0.00 & 1.00 & 1.69 \\
13 & 513.3 & 518.0 & 533 & 466 & 520 & 0.01 & 0.99 & 1.10 \\
14 & 541.3 & 537.0 & 570 & 529 & 551 & 0.02 & 0.98 & 1.05 \\
15 & 570.3 & 552.9 & 608 & 557 & 577 & 0.01 & 0.99 & 1.5 \\
16 & 631.1 & 628.8 & 667 & 627 & 640 & 0.00 & 1.00 & -0.4 \\
17 & 662.5 & 662.0 & 738 & 640 & 672 & 0.02 & 0.98 & 1.32 \\
18 & 769.8 & 727.1 & 821 & 716 & 773 & 0.01 & 0.99 & 1.57 \\
\hline
\end{tabular}

LDA calculation by Zhao and Vanderbilt (Ref. 10).

${ }^{\mathrm{b}}$ GGA calculation by Zhao and Vanderbilt (Ref. 10).

${ }^{\mathrm{c}}$ Experimental measurement by Arashi (Ref. 24).

${ }^{\mathrm{d}}$ Calculated from present experimental data.

${ }^{\text {e}}$ Room-temperature pressure data by Jayaraman et al. (Ref. 8).

mode Grüneisen parameters to decrease with mode frequency, related to the atom displacements in each mode. We divide the normal modes into two groups: oxygen-dominated modes and metal-dominated modes. From Fig. 2 it is apparent that the oxygen modes undergo more thermal softening than the metal modes. An especially interesting comparison is the softening of the number 2 and 3 modes. At $700 \mathrm{~K}$ the two modes are resolved and the absolute mode spacing increases at higher temperatures. The number 2 mode is composed of $95 \%$ oxygen whereas the number 3 mode is $75 \%$ oxygen.

The temperature dependence of the Raman peak widths provide further information about anharmonicity. ${ }^{25,26}$ The observed Lorentzian peak shapes are consistent with the expected energy broadening from shortened phonon lifetimes. In Fig. 3, a plot of the absolute broadening of normal modes demonstrates that the oxygen modes (with reasonable resolution across the measured range of temperatures) broaden faster than the metal modes, qualitatively consistent with the trend found for mode softening. (Note that in calculation of the linewidth, the number 2 and 3 peaks must be treated independently as the two peaks begin to soften at different rates, fitting both peaks with a single Lorentzian function would result in widening even if the individual peak widths remain unchanged.)

A useful perspective on the hafnia structure (Fig. 4) is that oxygen anions make up the structural framework of the crystal and the hafnium ions are located in the gaps between the oxygen ions. A more detailed view shows that the structure of hafnia contains layers of oxygen anions, with the remaining oxygen and hafnium ions inserted loosely between these layers. The oxygen layers lie approximately in the plane of the $b$ and $c$ axes, with the $b$ direction closer packed than the $c$ direction. Figure 4 depicts the ionic radius of oxygen

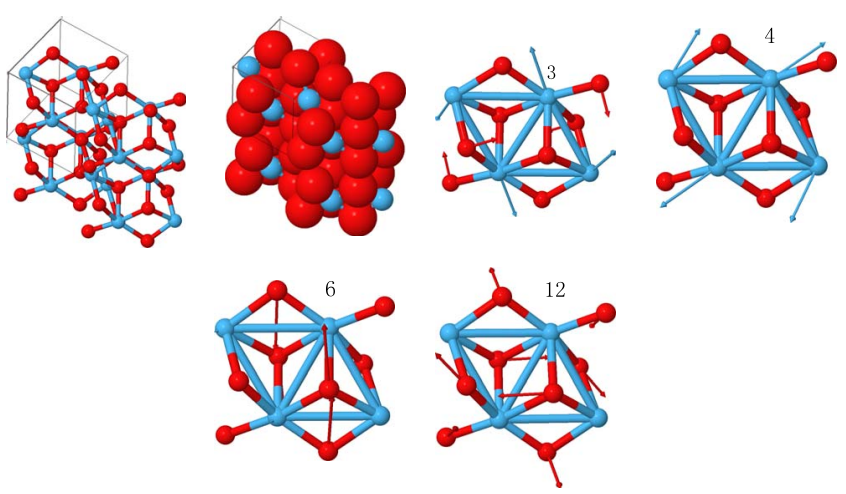

FIG. 4. (Color online) (Upper) Two views of the crystal structure of monoclinic hafnia. Red (light gray) and blue (dark gray) spheres denote oxygen and hafnium atoms, respectively. Left: skeletal; right: space-filled model, scaled for ionic radii. Note that the majority of the volume is composed of oxygen while hafnium fits loosely between oxygen planes. (Lower) Representative Ramanactive normal modes in one unit cell. Number 3 and 4 modes are hafnium dominated. Number 6 and 12 are oxygen dominated. These modes were well resolved through the whole range of temperatures. Lengths of arrows are proportional to the contribution of vibration from each atom. 
$\left(\begin{array}{ll}1.40 \AA \\ )\end{array}\right)$ as almost twice as big as that of hafnium $(0.78 \AA)$. Based on structure data from x-ray diffractometry, ${ }^{27}$ the oxygen-oxygen bonds have lengths of 2.51 or $2.64 \AA$ and hafnium-oxygen bonds have lengths of $1.98,2.22,2.17$, 2.23 , and $2.26 \AA$. As the lattice expands with temperature, the largest changes in atomic overlap occur for oxygen anions. Changes in this overlap are expected to affect the vibrational frequencies, especially for oxygen modes.

The analysis of individual normal modes, including the four shown in Fig. 4, offers more detailed information on the origin of nonharmonic behavior. The number 4 mode, representative of a hafnium-dominated mode, has hafnium cations moving into the cavity between three adjacent oxygen anions, in nearly parallel motion. The large size of the anions allow the cavity to impose few restrictions on the cations moving into it. The mode 4 is one of the most harmonic of all the Raman modes. For comparison, mode number 3 also is a metal-dominated mode where the metal cations move into cavities between the oxygen anions; however, half the oxygen anions in mode 3 are also moving, and they are moving in different planes with respect to each other. The contribution from oxygen to mode number 3 is larger (the partial DOS for mode 3 is $26 \% \mathrm{O}, 74 \% \mathrm{Hf}$ ) and it is less harmonic than mode number 4 (the partial DOS is $99 \% \mathrm{Hf}$ ).

In mode number 6 , half of the anions (the outer oxygen planes) are stationary while the remaining anions (the inner oxygen plane) are moving parallel to the $c$ axis-the nonstationary oxygen anions simply move into the space left by preceding ones. The result is that the oxygen-oxygen bond is largely unchanged during vibration. This mode is one of the most harmonic of all the oxygen-dominated modes. Mode number 12 is less harmonic. It involves relatively large changes in oxygen-oxygen bond lengths and the bending of oxygen-oxygen bonds. These four modes were chosen for their relative simple pattern of vibration. Other normal modes not shown in Fig. 4 provide similar correlations between oxygen-oxygen bond distortions and nonharmonic behavior. We find that the oxygen-dominated modes in hafnia are less harmonic than the metal-dominated modes. Furthermore, the modes involving a change in oxygen-oxygen bonding exhibit the most nonharmonic behavior.

Fig. 5 shows a parametric relationship between the peak broadening and the peak softening. A boundary exists between oxygen modes and metal modes, and all modes with non-negligible contributions from both elements fall close to the boundary. The larger nonharmonic effects are found for oxygen-dominated modes.

Phonon frequency shifts and broadenings have been analyzed by treating phonon-phonon interactions with perturbation theory. ${ }^{28}$ The analysis of Raman spectra is practical with the Klemens model, ${ }^{29}$ which considers how one optical phonon decays into two acoustical phonons. Analysis of this three-phonon process for decay of an optical phonon at the $\Gamma$ point is simplified because the two acoustic phonons must be of opposite wave vectors and matched in energy if they are on the same branch. (Equilibrium phonon populations are also assumed.) This approach has been extended to fourphonon processes ${ }^{30}$ but these simplifications are now less appropriate for describing the full physical picture. They do show that the temperature dependence becomes more

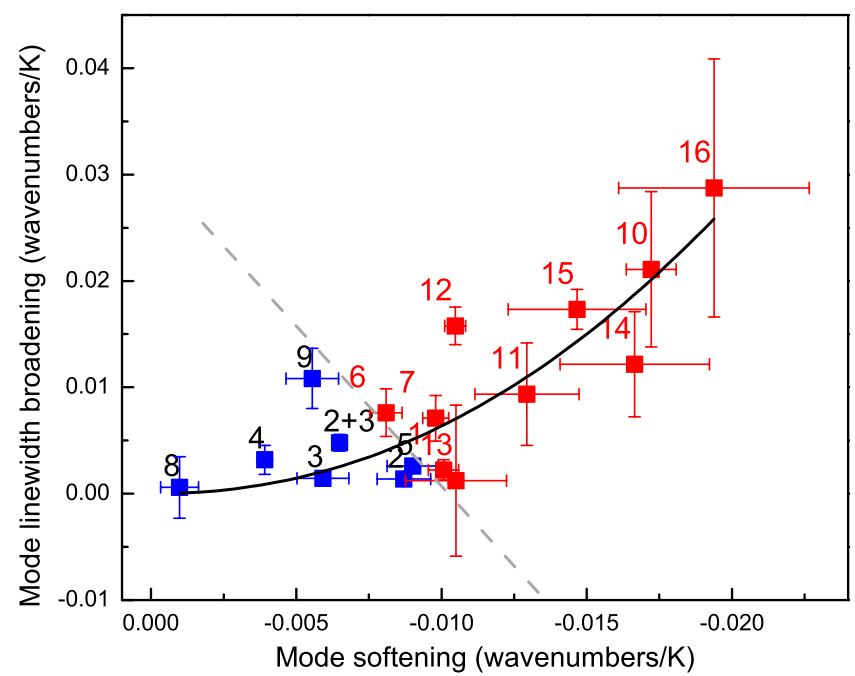

FIG. 5. (Color online) Broadening vs softening of Raman modes. Blue (dark gray) and red (light gray) squares are metal and oxygen modes, respectively. The dashed line is the boundary separating metal-dominated modes and oxygen-dominated modes. The solid line is a fitting to the points, showing that the relation between absolute linewidth broadening and absolute peak softening is of power 2.1, close to quadratic.

complex ${ }^{30}$ and the temperature-dependent phonon linewidth broadening $\Gamma_{j}$ of mode $j$ can be expected as

$$
\begin{aligned}
\Gamma_{j}(T)= & \Gamma_{j}(0)+A_{j}\left[1+\frac{2}{e^{\hbar \omega_{j}(0) / 2 k_{B} T}-1}\right] \\
& +B_{j}\left[1+\frac{3}{e^{\hbar \omega_{j}(0) / 3 k_{B} T}-1}+\frac{3}{\left(e^{\hbar \omega_{j}(0) / 3 k_{B} T}-1\right)^{2}}\right]
\end{aligned}
$$

where $\Gamma_{j}(0)$ is a constant; $A$ and $B$ are parameters for threephonon and four-phonon processes, respectively. At ambient or higher temperatures, the contributions from the two terms vary as $T$ and $T^{2}$, respectively. In the experimental data of Fig. 3, linewidth broadenings of the hafnia spectra are found to be linear with temperature so there is little contribution from the four-phonon process of Eq. (5) $(B=0)$. For different modes, the parameter $A$ ranged from 0.02 to $0.77 \mathrm{~cm}^{-1}$.

An analogous expression to Eq. (5) for the frequency shift at elevated temperature has been proposed ${ }^{30}$ but from experimental data at elevated temperature it is not practical to separate the anharmonic frequency shift from from the shift from quasiharmonic lattice dilation. Fortunately, pressure-induced shifts of Raman peaks were reported by Jayaraman et al. ${ }^{8}$ and these can be used to identify the quasiharmonic contribution. Their results are listed as Grüneien parameters in Table I. By comparing the pressure Grüneien parameters to the thermal Grüneien parameters, we find general agreement for all modes dominated by hafnium motions. On the other hand, there are major discrepancies for at least half of the oxygen modes and some have opposite signs. The deviation of our elevated temperature results from those of prior highpressure work again suggests that the oxygen-dominated modes are more anharmonic than the metal modes. Another 
result from perturbation theory is that the leading term for phonon broadening is the cubic term whereas the peak shift originates from the quartic term in first order and the cubic term in second order. ${ }^{31}$ With temperature, the metaldominated modes shift in frequency with comparatively little broadening. This could be primarily quasiharmonic behavior consistent with the high-pressure results. In contrast, for the oxygen-dominated modes, which show more broadening, the nonharmonic effects likely originate from the cubic term in the interatomic potential, consistent with the linearity with $T$ of the shifts and broadenings over the range of temperature in this study.

Nevertheless, the nonharmonic effects in hafnia at temperatures to $1100 \mathrm{~K}$ are relatively small and hafnia is generally quasiharmonic. The nonharmonic contribution is estimated to be about only $2 \times 10^{-3}[\mathrm{~J} /(\mathrm{mol} \mathrm{K})] / \mathrm{K}$ to the heat capacity, which is not large enough to explain the anomalously large slope of the heat capacity vs temperature curve for hafnia at these temperatures. ${ }^{13}$ Perhaps degrees of freedom other than phonons account for the excess heat capacity above $800 \mathrm{~K}$ or perhaps the heat-capacity results need to be checked.

\section{CONCLUSION}

Raman spectra were measured on monoclinic $\mathrm{HfO}_{2}$ at elevated temperatures up to $1100 \mathrm{~K}$. The peaks in the spectra were assigned to vibrational modes obtained by shell-model calculations, which provided the individual atom polariza- tions in each mode. The thermal softening and broadening of the Raman peaks were obtained from the measured spectra and correlated with the individual atom displacements in each mode. The oxygen-dominated modes were found to be more anharmonic than the hafnium-dominated modes. The least harmonic modes were those with the largest distortions of oxygen-oxygen bond distances (as opposed to oxygenhafnium or hafnium-hafnium). Hafnium-dominated modes showed relatively little peak broadening for the peak shift, consistent with an approximately quasiharmonic behavior, having thermal Grüneisen parameters that were more consistent with previous high-pressure results than those for oxygen-dominated modes. The oxygen-dominated modes showed more peak broadening with peak shift, indicating a relatively large role for the first-order cubic term in the anharmonic behavior. Very little contribution from four-phonon processes is found by fitting perturbation theory results. Peak shifts and peak broadenings, two strong indicators of the nonharmonicity of Raman modes, were quadratically correlated within the measured range of temperatures. The nonharmonic peak shifts in this temperature range are not large enough to account for the large previously reported heat capacity of hafnia above $800 \mathrm{~K}$.

\section{ACKNOWLEDGMENTS}

We thank M. S. Lucas for help with the furnace design and M. G. Kresch for discussions. This work was supported by the Department of Energy through Basic Energy Sciences under Grant No. DE-FG02-03ER46055.

*lichen@ caltech.edu

${ }^{1}$ G. D. Wilk, R. M. Wallace, and J. M. Anthony, J. Appl. Phys. 89, 5243 (2001).

${ }^{2}$ E. P. Gusev, E. Cartier, D. A. Buchanan, M. Gribelyuk, M. Copel, H. Okorn-Schmidt, and C. D'Emic, Microelectron. Eng. 59, 341 (2001).

${ }^{3}$ J. Wang, H. P. Li, and R. Stevens, J. Mater. Sci. 27, 5397 (1992).

${ }^{4}$ P. E. Quintard, P. Barbéris, A. P. Mirgorodsky, and T. MerleMéjean, J. Am. Ceram. Soc. 85, 1745 (2002).

${ }^{5}$ R. Ruh and P. W. R. Corfield, J. Am. Ceram. Soc. 53, 126 (1970).

${ }^{6}$ C. Carlone, Phys. Rev. B 45, 2079 (1992).

${ }^{7}$ E. Anastassakis, B. Papanicolaou, and I. M. Asher, J. Phys. Chem. Solids 36, 667 (1975)

${ }^{8}$ A. Jayaraman, S. Y. Wang, S. K. Sharma, and L. C. Ming, Phys. Rev. B 48, 9205 (1993).

${ }^{9}$ G. A. Kourouklis and E. Liarokapis, J. Am. Ceram. Soc. 74, 520 (1991).

${ }^{10}$ X. Zhao and D. Vanderbilt, Phys. Rev. B 65, 233106 (2002).

${ }^{11}$ A. A. Demkov, Phys. Status Solidi B 226, 57 (2001).

${ }^{12}$ G. Morell, W. Pérez, E. Ching-Prado, and R. S. Katiyar, Phys. Rev. B 53, 5388 (1996).

${ }^{13}$ C. D. Pears, Southern Res. Inst., USAF ASD-TDR-62-765, pp. 1-420 (1962).

${ }^{14}$ C. Wang, M. Zinkevich, and F. Aldinger, J. Am. Ceram. Soc. 89,

3751 (2006).

${ }^{15}$ L. M. Bollinger, S. P. Harris, C. T. Hibdon, and C. O. Muehlhause, Phys. Rev. 92, 1527 (1953).

${ }^{16}$ Alfa Aesar, Ward Hill, MA 01835.

${ }^{17}$ Quanta-Ray Lab Series.

${ }^{18}$ Semrock Stopline E-grade single-notch filter.

${ }^{19}$ Princeton Instruments Acton Series $500 \mathrm{~mm}$.

${ }^{20}$ Princeton Instruments PIXIS 400B.

${ }^{21}$ J. D. Gale and A. L. Rohl, Mol. Simul. 29, 291 (2003).

${ }^{22}$ G. V. Lewis and C. R. A. Catlow, J. Phys. C 18, 1149 (1985).

${ }^{23}$ C. F. Grain and W. J. Campbell, U. S. Bur. Mines, Report No. BM-RI-5982, 1962.

${ }^{24}$ H. Arashi, J. Am. Ceram. Soc. 75, 844 (1992).

${ }^{25}$ J. Serrano, F. J. Manjón, A. H. Romero, F. Widulle, R. Lauck, and M. Cardona, Phys. Rev. Lett. 90, 055510 (2003).

${ }^{26}$ J. Serrano, F. Widulle, A. Romero, A. Rubio, R. Lauck, and M. Cardona, Phys. Status Solidi B 235, 260 (2003).

${ }^{27}$ D. M. Adams, S. Leonard, D. R. Russell, and R. J. Cernik, J. Phys. Chem. Solids 52, 1181 (1991).

${ }^{28}$ A. A. Maradudin and A. E. Fein, Phys. Rev. 128, 2589 (1962).

${ }^{29}$ P. G. Klemens, Phys. Rev. 148, 845 (1966).

${ }^{30}$ M. Balkanski, R. F. Wallis, and E. Haro, Phys. Rev. B 28, 1928 (1983).

${ }^{31}$ M. Zoli, J. Phys.: Condens. Matter 3, 6249 (1991). 